\title{
Machinery signal separation using non-negative matrix factorization with real mixing
}

\author{
Anindita Adikaputri Vinaya ${ }^{1}$, Sefri Yulianto ${ }^{2}$, Qurrotin A'yunina Maulida Okta Arifianti ${ }^{3}$, \\ Dhany Arifianto ${ }^{4}$, Aulia Siti Aisjah ${ }^{5}$ \\ ${ }^{1,2,3}$ Department of Engineering Management, Universitas Internasional Semen Indonesia, Indonesia \\ ${ }^{4,5}$ Department of Engineering Physics, Institut Teknologi Sepuluh Nopember, Indonesia
}

\section{Article Info}

Article history:

Received Aug 16, 2019

Revised Oct 18, 2019

Accepted Dec 11, 2019

\section{Keywords:}

Fundamental frequency

Machinery signal

NMF

Real mixing

\begin{abstract}
A big challenge in detecting damage occurs when the sound of a machine mixes with the sound of another machine. This paper proposes the separation of mixed acoustic signals using Non-negative Matrix Factorization (NMF) method for fault diagnosis. The NMF method is an effective solution for finding hidden parameters when the number of observations obtained by the sensor is less than the number of sources. The real mixing process is done by placing two microphones in front of the machine. Two microphones will be used as sensors to capture a mixture of four machinery signals. Performance testing of signal separation is done by comparing baseline signals with estimated signals through the mean log spectral distance (LSD) and the mean square error (MSE). The smallest spectral distance between the estimated signal and the baseline signal is found in $\hat{\mathrm{S}} 2$ with an average LSD of 1.26. The estimated signal $\hat{\mathrm{S}} 2$ is the closest to the baseline signal with MSE of $1.15 \times 10-2$. The pattern of bearing damage in the male screw compressor can be identified from the spectrum of estimated signal through harmonic frequencies as in the estimated signal $\hat{\mathrm{S}} 3$ which is seen at $11 \mathrm{x}$ fundamental frequency, $12 \mathrm{x}$ fundamental frequency, $15 \mathrm{x}$ fundamental frequency, and 16x fundamental frequency.
\end{abstract}

This is an open access article under the CC BY-SA license.

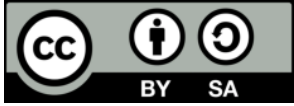

\section{Corresponding Author:}

Anindita Adikaputri Vinaya,

Department of Engineering Management,

Universitas Internasional Semen Indonesia,

Jalan Veteran, Gresik, 61122, East Java, Indonesia

Email: anindita.vinaya@uisi.ac.id

\section{INTRODUCTION}

The production process in industry can not be separated from the use of machines. One of the important machinery and widely used in the industry is rotating machinery. Monitoring of machine conditions is very important to do in the industry [1]. The acoustic signal can be used to analyze the condition of the machinery beside the vibration signal. The characteristics of each machine can be distinguished from the pattern formed [2-4]. Mixing of one signal with another signal is a common condition which can make destruction of the original signal. Separation of machinery signals is needed to get a signal that approaches the original signal $[1,5]$. Blind Source Separation (BSS) is a technique to separate a mixed signal in a "blind" state. In this case the "blind" is not knowing the mixing signal, only knowing the mixed signal [6]. In the BSS technique, several methods can be used to separate acoustic emission signals. One of them is Independent Component Analysis (ICA). By an assumption on the statistical independence between the source signals, BSS has been successfully tackled by ICA, which has become a standard statistical 
method for separation problems [7, 8]. The ICA derivative method, FastICA, has been used by Ghita et al. to separate signals both in artificial mixing and in actual mixing. Mixed signals from 4 human voices were obtained from 4 sensors. Each source signal in the two blends carried out was reconstructed successfully [8]. The same method is also used by Farhat et al. to separate gear signals. Healthy and damaged wheel components were identified [5]. Ajami et al. used ICA to identify the condition of a real turbine system. The PCA approach is also used to help reduce the dimensions of the data obtained. The results showed that the method was effective in identifying damage [9]. The combination of ICA and SVM was proposed by Ji and Zhang in the diagnosis and classification of engine damage [10]. The combination of ICA and ensemble Empirical Mode Decomposition (EMD) methods succeeded in separating multiple failures on bearings with different speed variations [11]. The ICA method can generally separate signals well with fast computing time when the number of observations is equal or more than the number of sources $[12,13]$.

One method that can be used to find out hidden parameters when the number of observations obtained by the sensor is less than the number of sources is non-negative matrix factorization (NMF). This method was proposed by Lee and Seung where a guarantee of signal independence such as the ICA method is not needed [6]. The NMF method has been used to separate audio signals [14, 15], vibration signals [16, 17], EEG signals [18], and others. The signal mixing process has been done in both artificial mixing and real mixing [8]. NMF is used by Liang et al. to decompose spectral and frequency bands that indicate bearing damage through vibration signals. Verification of the results of the study was carried out with a fault machine simulator with an accelerometer as a sensor [16]. Separation of machine signals with real mixing is done by Miao et al. using blind source separation based on second order statistics [19].

The research object used is two-span rotors. The mixed signal is obtained from 3 vibration sensors. Random noise can be separated in that study [19]. Wodecki et al. used the NMF method to identify damage to the gear box on the conveyor belt at the real plant. The spectrogram of the vibration signal is used in the analysis of the gear box fault [17]. The NMF method can also be combined with neural networks[20] and $\mathrm{K}$ nearest neighbors[21] for fault diagnosis. The NMF method added by Jiang et al. in the incremental broad learning approach (IBL) is more effective than without the addition of NMF in diagnosing damage to a three-phase induction motor[22]. The NMF method will be adopted to separate the machinery signals in this study. The real mixing process is done by placing two microphones in front of the machine. The microphone will be used as a sensor to capture a mixture of machinery signals. Signal representation in the frequency domain will be used in signal analysis.

\section{RESEARCH METHOD}

\subsection{Blind source separation}

Blind Source Separation is a mixed signal separation technique to predict the original signal in an unknown condition of the mixing process or mixing matrix A. The information obtained is only a mixed signal $\mathrm{X}$. This method of signal separation aims to rearrange the original signal $\mathrm{S}$ which has been mixed $[7,12]$. Here is an equation of the BSS method:

$$
x(t)=\sum_{i=1}^{n} A(\tau) \cdot S(t-\tau)
$$

Where $\mathrm{S}$ consist of $\left[\mathrm{S}_{1}, \mathrm{~S}_{2}, \ldots . \mathrm{Sl}\right]^{\mathrm{T}}$. $\mathrm{J}$ is the column vector matrix $1 \times 1$ (collection of sources), $\mathrm{x}$ consists of $\left[\mathrm{x}_{1}, \mathrm{x}_{2}, \ldots \mathrm{x}_{1}\right]^{\mathrm{T}}$ is sets of 1 observed signals, $\mathrm{A}$ is $\mathrm{m} \mathrm{x} \mathrm{n}$ matrix. $\mathrm{m}$ is the number of sensors and it contains mixing coefficients $b_{j}(t)$, and $\mathrm{n}$ is the number of sources. Equation 1 is a convolution equation in the time domain. We should convert it into the frequency domain using a Short Time Fourier Transform (STFT) to increase sparsity through equation [13]:

$$
\operatorname{STFT}\left(t^{\prime}, \omega\right)=\int\left[x(t) \cdot w\left(t-t^{\prime}\right)\right] \cdot e^{-j \omega t} d t
$$

Where $w\left(t-t^{\prime}\right)$ is window function. In this study, sine window function is used with an overlap of $50 \%$ and the length of the STFT window is 1024 . Signals will be converted into the frequency domain by using STFT in which each frequency band can be known independently. Equation 1 can be written as follows:

$$
\mathrm{x}(\omega)=\sum_{\mathrm{i}=1}^{\mathrm{n}} A(\omega) \cdot \mathrm{S}(\omega)
$$




\subsection{Non-negative matrix factorization}

In the BSS technique, several methods can be used to separate acoustic emission signals [12, 23].

One of them is non-negative matrix factorization (NMF). NMF is an effective matrix factorization method for decomposing multivariate data under the boundaries of non-negative components[12]. NMF aims to factor the algorithm $[\mathrm{V}] \approx[\mathrm{W}] \times[\mathrm{H}]$ so that it can be reduced in dimensions. $\mathrm{V}$ is a mixed signal that will be decomposed into a mixing matrix $\mathrm{W}$ and sound source $\mathrm{H}$. In the signal separation process, the matrix [V] represents magnitude or spectral power of the signal. The matrices $\mathrm{W}$ and $\mathrm{H}$ are decompositions of the spectrogram $\left|S_{j}\right|^{2} \approx W_{j} H_{j}[14,15]$. The matrices $\mathrm{W}$ and $\mathrm{H}$ are randomly initialized which have non-negative components. The problem of minimization of factoring $\mathrm{W}$ and $\mathrm{H}$ will be overcome by minimizing divergence:

$$
\min _{W, H \geq 0} D(V \mid W H)
$$

In minimizing equation 4, the Itakura Saito divergence where $D(V \mid W H)=D(V \mid \widehat{V})$ is used. The Itakura-Saito equation had a statistical solution to the problem of minimizing the non-negative matrix [14].

$$
\begin{aligned}
& d_{I S}(x \mid y)=\frac{x}{y}-\log \frac{x}{y}-1 \\
& C(\theta)=\sum_{m, \omega} d_{I S}\left(\left|x_{m, \omega}\right|^{2} \hat{v}_{m, \omega}\right)
\end{aligned}
$$

Where $\mathrm{x}, \mathrm{y} \geq 0$ and $d_{I S}(x \mid y)$ is Itakura Saito based scalar divergence which measures the closeness between two signals. The cost function based on Itakura Saito is written in equation 6, where $\theta$ is $\mathrm{W}$, $\mathrm{H}$ paramaters and $\hat{v}_{m, \omega}$ is the sum of variance. The likelihood distribution is known to be more maximal with the iterative process. One method that can be used is the multiplicative update algorithm. The iterative process carried out by this method is a process for updating each parameter. The goal is to bring up hidden parameters in each channel.

The likelihood estimation of $\mathrm{W}$ and $\mathrm{H}$ is based on the Itakura Saito divergence criteria which is derived from $\beta$ divergence when $\beta=0[12] . V$ is an input in equation 7 and equation 8 . The matrix $\mathrm{W}$ and $\mathrm{H}$ will be initialized with non-negative values, where $\widehat{V}=\mathrm{W} . \mathrm{H}$. When initializing $\mathrm{W}$ and $\mathrm{H}$ are entered in the equations, the new $\mathrm{W}$ value and $\mathrm{H}$ value will be obtained. The $\mathrm{W}$ and $\mathrm{H}$ matrices will be updated so that $[V] \approx[\mathrm{W}] \times[\mathrm{H}]$. The matrices $\mathrm{W}$ and $\mathrm{H}$ will be normalized then re-iteration step will be applied until reach convergence[15]. In this study, the number of iteration used is 500. Wiener filter based on minimum mean square error criteria is applied to reconstruct the source signal, after the best likelihood the parameters have been found through the iteration process above. Inverse STFT is used to obtain the source signal in the time domain.

$$
\begin{aligned}
\text { Update } W & W_{n} \leftarrow W_{n} \cdot \frac{\left(\hat{V}_{m}^{-2} \cdot V_{m}\right) H_{n}^{T}}{\widehat{V}_{m}^{-1} H_{n}^{T}} \\
\text { Update } H & H_{n} \leftarrow H_{n} \cdot \frac{\left(\widehat{V}_{m}^{-2} \cdot V_{m}\right) W_{n}^{T}}{\nabla_{m}^{-1} W_{n}^{T}}
\end{aligned}
$$

The signal obtained will be transformed into the frequency domain to analyze the spectrum. The spectrum is represented by amplitude as a function of frequency. The frequency spectrum is used to determine the condition of the engine because the analysis of signals in the time domain is more difficult than the analysis of signals in the frequency domain $[1,24]$. Fast Fourier Transform(FFT) will be used to get the frequency spectrum. The flowchart of the machinery signal separation process can be seen in Figure 1.

\subsection{Experimental setup}

A compressor is a device for compressing gas fluid through volume reduction. The compressor functions to increase fluid compressibility. GA 37 Rotary screw compressor is an object in this study. The maximum capacity of this compressor engine is 10 Bar with $37 \mathrm{~kW}$ motor power and 3000 RPM rotational speed. In this study, four parts of the compressor machinery will be used as sound sources. $\mathrm{S}_{1}$ is the signal source located on the motor that is far from the screw compressor, $S_{2}$ is the signal source located on a motor that is close to the screw compressor, $\mathrm{S}_{3}$ is the signal source located on a male compressor screw 
that is close to the motor, and $\mathrm{S}_{4}$ is the signal source located on a male compressor screw that is far from the motor. The location of the source can be seen in Figure 2.

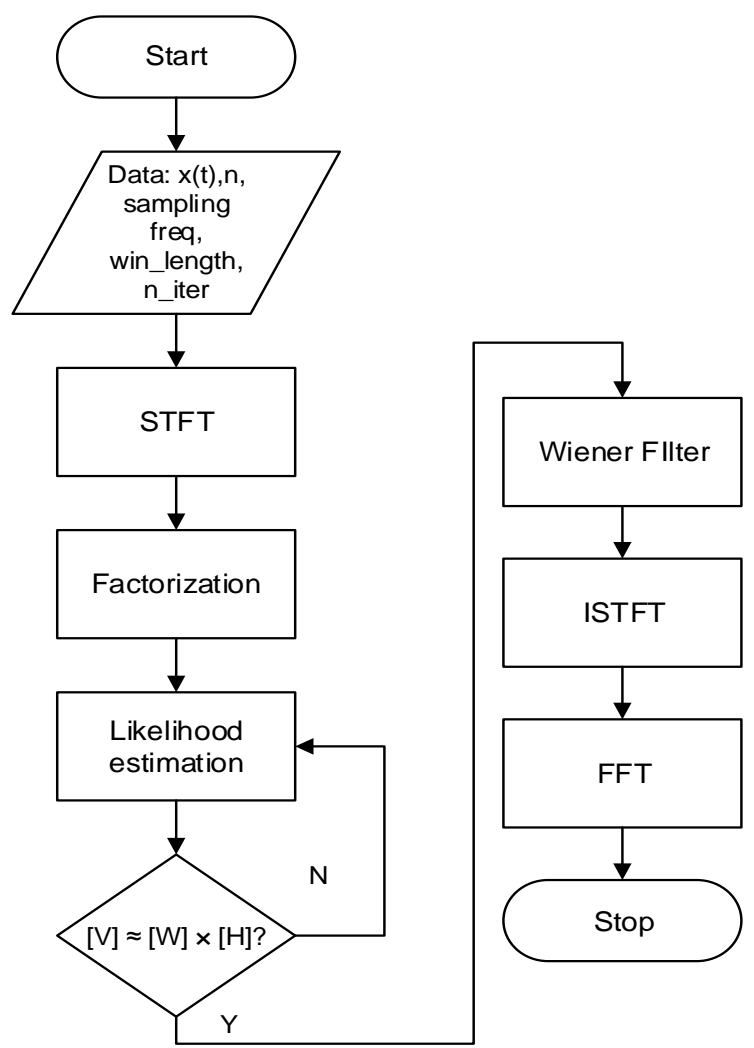

Figure 1. Flowchart of mixed signal separation

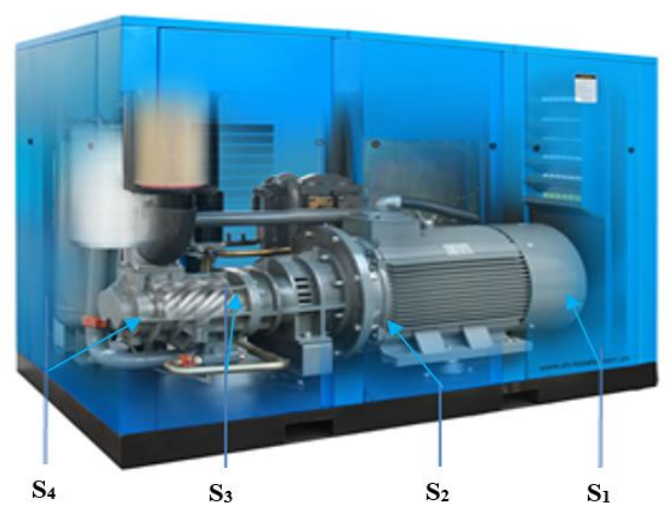

Figure 2. Simplified representasion of sound source from the compressor machinery used in this study

The data collection is done in two stages, i.e., baseline signal recording and mixed signal recording. The recording time is five second. At the baseline signal recording, Behringer XM1800S cardioid type microphone is placed at a distance of $5 \mathrm{~cm}$ from the sound source. It is assumed that at that distance, the sound signal obtained will approach the engine vibration signal on that side. At mixed signal recording, two microphones are placed at $90 \mathrm{~cm}$ from the sound sources with a distance between microphones is $15 \mathrm{~cm}$ as shown in Figure 3. In this study, Behringer XM1800S microphone used as a sound sensor is connected with an XLR cable, while the interface used as an analog to digital data converter is USB Audio Interface. The baseline signal recording used $44100 \mathrm{~Hz}$ as a sampling frequency with 220500 samples. The sampling frequency adjusts to the minimum sampling frequency of the USB audio interface.The downsampling will be applied from $44100 \mathrm{~Hz}$ to $8000 \mathrm{~Hz}$ with the amount of 40000 samples, to accelerate the processing of data. 


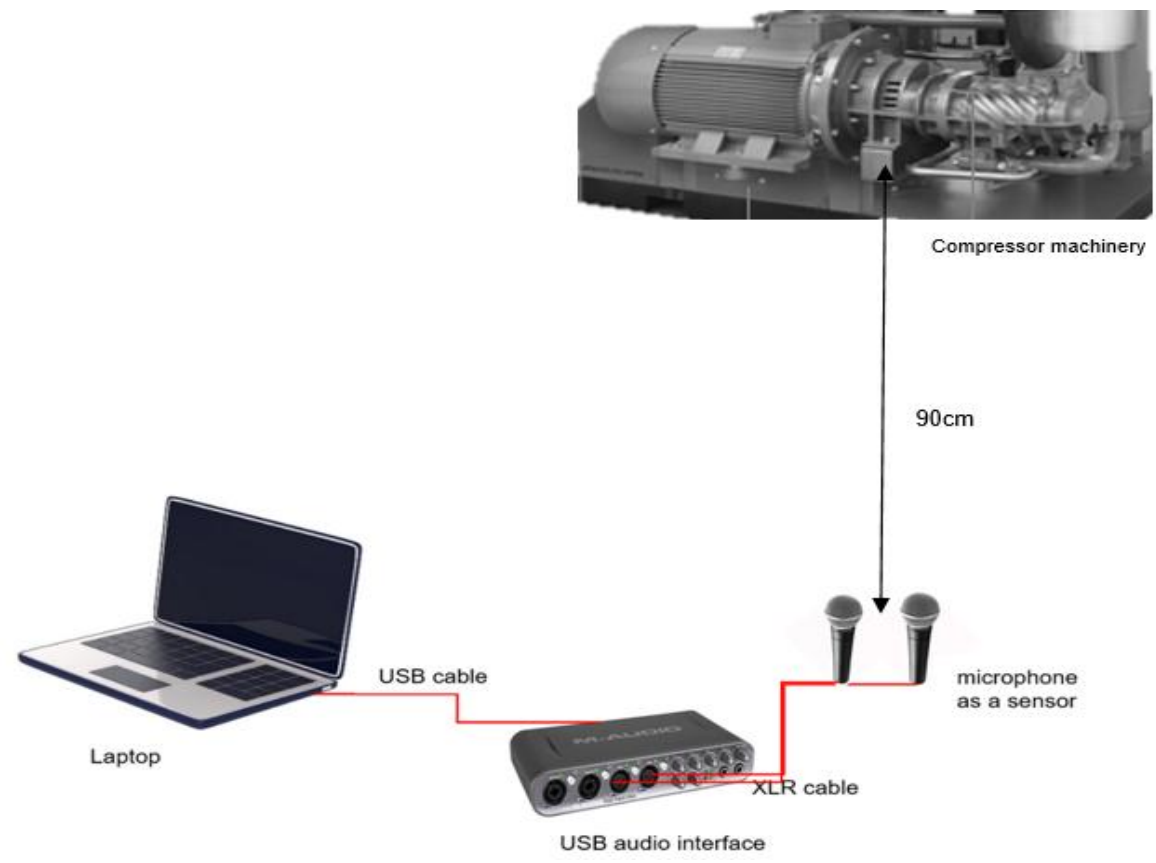

Figure 3. Components used in signal recording with real mixing

\section{RESULTS AND DISCUSSION}

The recording results from the baseline signal are shown in Figure 4. The spectrum of $S_{1}$ to $S_{4}$ have different characteristics. The lowest peak amplitude value of the others is owned by spectrum $\mathrm{S} 1$ which is $2.9 \times 10^{-2}$ at $594 \mathrm{~Hz}$ or about $12 \mathrm{x}$ the fundamental frequency of the machine. The fundamental frequency is obtained from the rotating speed of machine which is around 3000RPM. Based on the baseline signal spectrum, the fundamental frequency is $49.56 \mathrm{~Hz}$. The peak amplitude of $S_{2}$ and $S_{4}$ is also located at $12 x$ the fundamental frequency of the machine but in $S_{4}$ there is a high amplitude of $4.3 \times 10^{-2}$ which is located at $543.9 \mathrm{~Hz}$ or around $11 \mathrm{x}$ the fundamental frequency. The high amplitude possessed in the $\mathrm{S}_{3}$ spectrum are $6.1 \times 10^{-2}$ and $4.4 \times 10^{-2}$ which are located at $790.9 \mathrm{~Hz}$ or $16 \mathrm{x}$ the fundamental frequency and $642.6 \mathrm{~Hz}$ or $13 \mathrm{x}$ the fundamental frequency.

The mixed signal will go through a signal separation process using the proposed method. Four estimated signals will be generated from the separation process, where $\hat{S}_{1}$ is an estimated signal from $S_{1}$, $\hat{\mathrm{S}}_{2}$ is an estimated signal from $\mathrm{S}_{2}, \hat{\mathrm{S}}_{3}$ is an estimated signal from $\mathrm{S}_{3}$, and $\hat{\mathrm{S}}_{4}$ is an estimated signal from $\mathrm{S}_{4}$. The estimated signals are shown in Figure 5.The fundamental frequency of the estimated signals is $49.56 \mathrm{~Hz}$. This frequency corresponds to the fundamental frequency of the baseline signals. The estimated signal $\hat{\mathrm{S}} 1$ has a peak amplitude of $4.8 \times 10^{-3}$ at $594.7 \mathrm{~Hz}$ where the peak amplitude of the baseline signal $\mathrm{S}_{1}$ lies at $594 \mathrm{~Hz}$ or around $12 \mathrm{x}$ the fundamental frequency. Based on the overall spectrum estimation, the peak amplitude of the signal $\hat{S}_{1}$ is the smallest compared to other signals where the baseline signal $S_{1}$ also has the same characteristics. The estimated signal $\hat{\mathrm{S}}_{2}$ has a high amplitude at $11 \mathrm{x}$ the fundamental frequency and $12 \mathrm{x}$ the fundamental frequency while the high amplitude of baseline signal $S_{2}$ is located at $12 x$ the fundamental frequency and $13 x$ the fundamental frequency. Harmonic frequencies with high amplitude of the estimated signal $\hat{S}_{3}$ are seen at $11 x$ the fundamental frequency, $12 x$ the fundamental frequency, $15 x$ the fundamental frequency and $16 \mathrm{x}$ the fundamental frequency, while the baseline signal $\mathrm{S}_{3}$ is located at $12 \mathrm{x}$ the fundamental frequency, $13 \mathrm{x}$ the fundamental frequency, $15 \mathrm{x}$ the fundamental frequency and $16 \mathrm{x}$ the fundamental frequency. The peak amplitude of the estimated signal $\hat{S}_{4}$ is at $12 \mathrm{x}$ the fundamental frequency. It is followed by the emergence of the amplitude at $13 x$ the fundamental frequency where it is aligned with the $\mathrm{S}_{4}$ baseline signal. Based on the results of the separation, there are differences in the location of the peak amplitude and amplitude shift at some high frequencies but the spectrum patterns still have the characteristics and compatibility with the baseline signals. In this study, the results of the separation were evaluated through the mean log spectral distance (LSD) and the mean square error (MSE) shown in Table 1. The lowest LSD is found in $\hat{\mathrm{S}}_{2}$ which is 1.26 and the lowest MSE value is $1.15 \times 10^{-2}$. It shows that the estimated signal $\hat{\mathrm{S}}_{2}$ is closest to the baseline signal $\mathrm{S}_{2}$. 

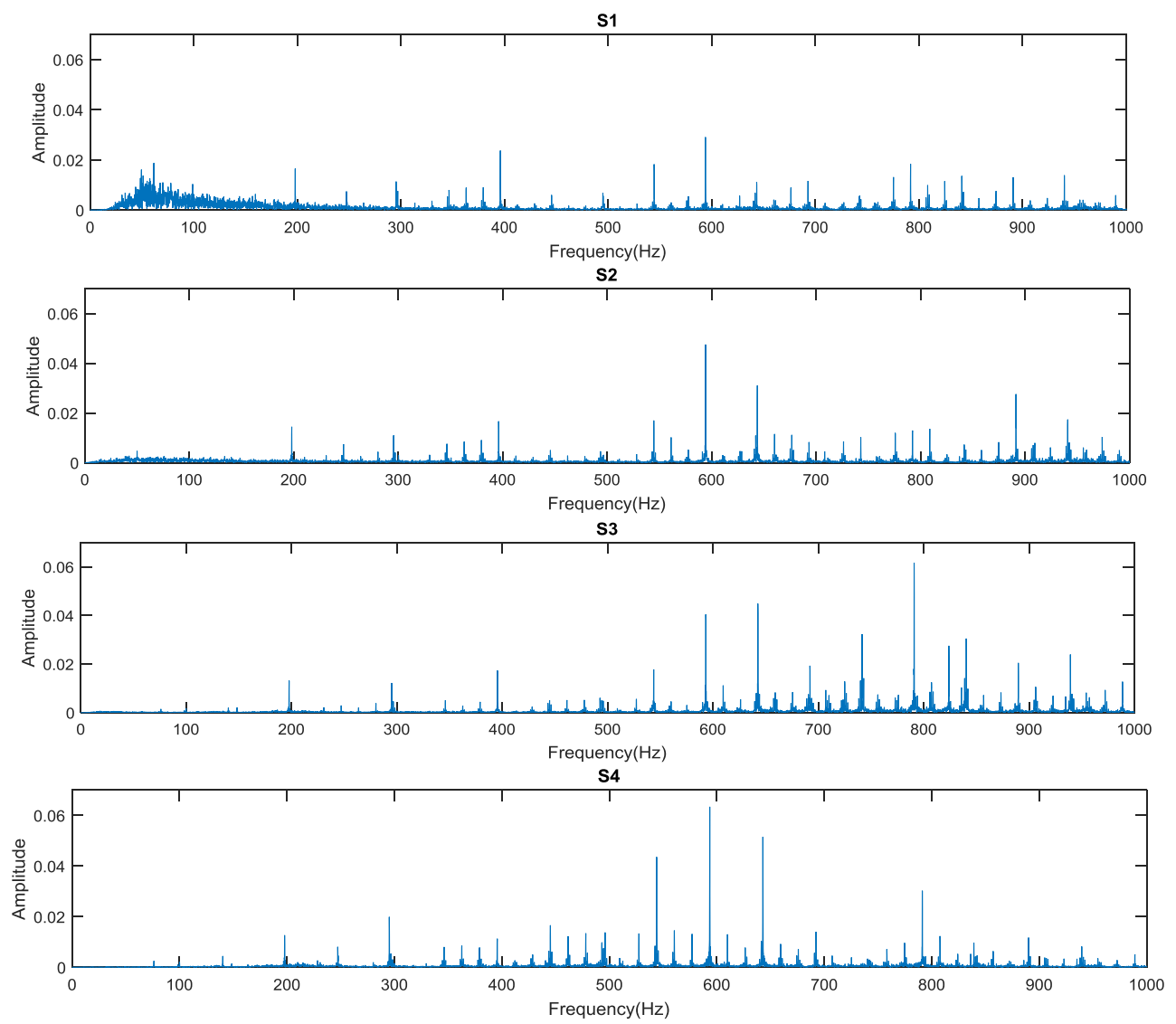

Figure 4. The results of baseline signal recording in frequency domain
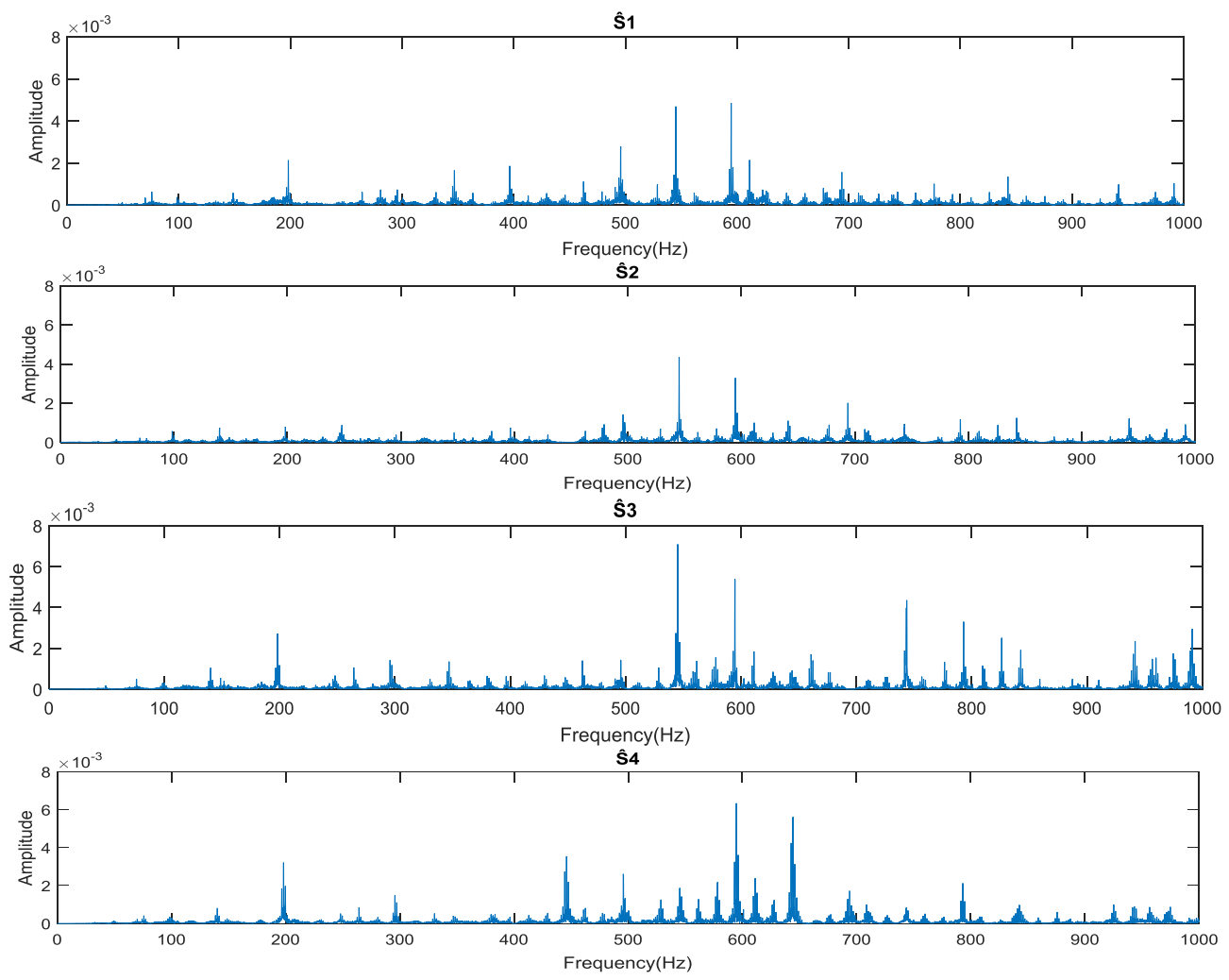

Figure 5. The results of separation process in frequency domain 
Table 1. The performance of separation process

\begin{tabular}{ccc}
\hline Signal & $\overline{\mathrm{LSD}}$ & $\overline{\mathrm{MSE}}$ \\
\hline$\hat{\mathrm{S}}_{1}$ & 1.39 & $1.34 \times 10^{-2}$ \\
$\hat{\mathrm{S}}_{2}$ & 1.26 & $1.15 \times 10^{-2}$ \\
$\hat{\mathrm{S}}_{3}$ & 1.33 & $1.93 \times 10^{-2}$ \\
\hline
\end{tabular}

The vibration signal from the accelerometer is used to verify the machinery condition in this study. Based on reports from vibration analysis experts in the industry [25], the compressor parts that have significant bearing damage are $S_{3}$ and $S_{4}$. One of the results of vibration analysis can be seen in Figure 6.

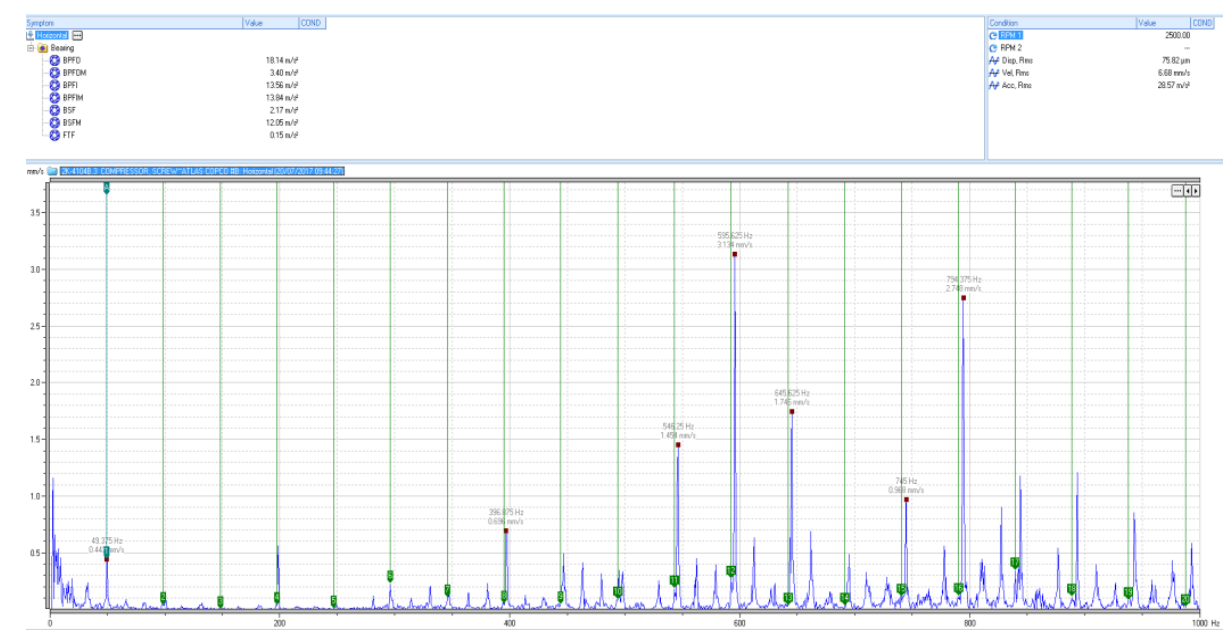

Figure 6. Vibration analysis for $\mathrm{S}_{3}$ with bearing fault

In the vibrational spectrum, the fundamental frequency is $49.375 \mathrm{~Hz}$. This bearing damage location can be seen from the appearance of high amplitude on the harmonic frequencies of bearing defects such as $546.25 \mathrm{~Hz}$ or around $11 \mathrm{x}$ the fundamental frequency, $595.625 \mathrm{~Hz}$ or around $12 \mathrm{x}$ the fundamental frequency, $645.625 \mathrm{~Hz}$ or around $13 \mathrm{x}$ the fundamental frequency, $741 \mathrm{~Hz}$ or around $15 \mathrm{x}$ the fundamental frequency and $794.75 \mathrm{~Hz}$ or around $16 \mathrm{x}$ the fundamental frequency. Vibration on $S_{3}$ and $S_{4}$ are higher than other machines, which are $6.68 \mathrm{~mm} / \mathrm{s}$ and $9.36 \mathrm{~mm} / \mathrm{s}$. Indications of significant damage have not been found in $S_{1}$ and $S_{2}$. Vibration on $S_{1}$ and $S_{2}$ is lower than other machines, which are $3.72 \mathrm{~mm} / \mathrm{s}$ and $3.50 \mathrm{~mm} / \mathrm{s}$. Based on the ISO 10816-vibration severity standard, these values are still within tolerable limits while the vibrations on S3 and S4 have entered into unacceptable conditions and required bearing maintenance.

The pattern of bearing damage from the separation of the signals shown in Figure 7. There are differences in the location of the peak amplitude in Figure 6 and Figure 7. In the vibrational spectrum $S_{3}$, the peak amplitude of $3.13 \mathrm{~mm} / \mathrm{s}$ lies in $595.625 \mathrm{~Hz}$ or around $12 \mathrm{x}$ the fundamental frequency while the peak amplitude on the acoustic spectrum $\mathrm{S}_{3}$ lies in $545.2 \mathrm{~Hz}$ or around $11 \mathrm{x}$ the fundamental frequency. In the acoustic spectrum of $\hat{\mathrm{S}}_{3}$, the fundamental frequency is $49.56 \mathrm{~Hz}$. Bearing defect on $\mathrm{S}_{3}$ can still be known based on harmonic frequencies, such as $545.2 \mathrm{~Hz}$ or around $11 \mathrm{x}$ the fundamental frequency, $594.7 \mathrm{~Hz}$ or around $12 \mathrm{x}$ the fundamental frequency, $743.7 \mathrm{~Hz}$ or around $15 \mathrm{x}$ the fundamental frequency, and $793 \mathrm{~Hz}$ or around $16 \mathrm{x}$ the fundamental frequency.

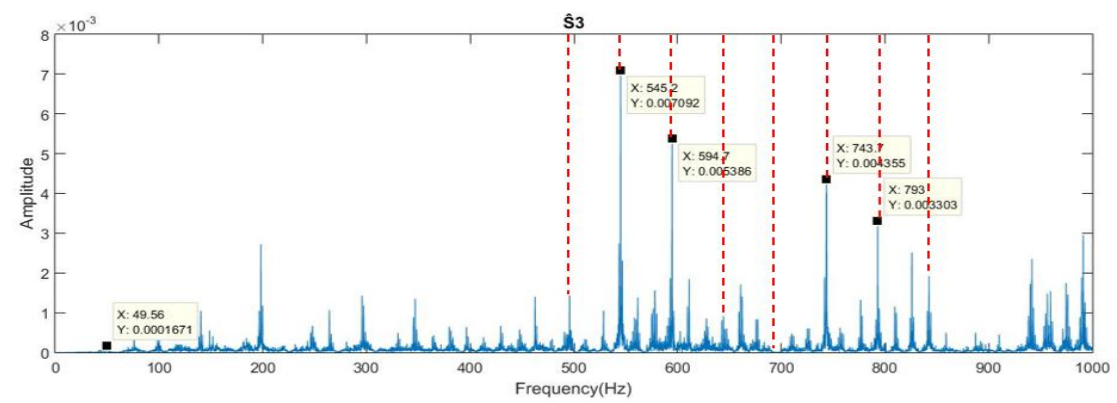

Figure 7. Acousic spectrum of $\hat{\mathrm{S}}_{3}$ 


\section{CONCLUSION}

Separation of mixed signals from four machines based on NMF method with real mixing was done in this study. The estimated signal $\hat{\mathrm{S}}_{2}$ is the closest to the baseline signal with the LSD of 1.26 and the MSE of $1.15 \times 10^{-2}$. Acoustic signals from each part of the compressor engine have different characteristics. Based on experts in the industry, bearing damage is shown in male screw compressors $\mathrm{S}_{3}$ and $\mathrm{S}_{4}$ through high amplitudes and harmonic frequency patterns in both vibrational and acoustic signals. Based on the ISO 10816-vibration severity standard, maintenance and preparation of bearing reserves are required. The severity can be known from the vibration signal through its velocity. In this study, acoustic signals are used to determine the location of damage. The acoustic signal has not been used to determine the severity. Some experiments are needed to analyze the severity which will be the focus of further research. Based on the separation results, a shift in the location of the high amplitude occurs in some estimated signals. Nevertheless, the pattern of the spectrum of each signal and an indication of the damage can be identified. In future research, differences in bearing patterns such as outer race defects, inner race defects, and others will also be analyzed

\section{ACKNOWLEDGEMENTS}

We thank the Direktorat Riset dan Pengabdian kepada Masyarakat (DRPM), Ditjen Penguatan Riset dan Pengembangan, Ministry of Research, Technology and Higher Education which has funded this research (Research Collaboration between Universities) with contract number : 071/SP2H/LT/MONO/L7/2019.

\section{REFERENCES}

[1] Azeem, N., Yuan, X., Raza, H., \& Urooj, I., "Experimental condition monitoring for the detection of misaligned and cracked shafts by order analysis," Advances in Mechanical Engineering, vol. 11, no 5, pp. 1-11, 2019.

[2] Sumarno, L, Tjendro, Widyastuti, W, Wihadi, D., "Water pump mechanical faults display at various frequency resolutions.," Telkomnika, vol. 12, no.1, pp. 97-106, 2014

[3] Wang, Y. F., \& Peng, X. Y., "Fault Diagnosis of Reciprocating Compressor Valve Using Acoustic Emission," 2012 International Mechanical Engineering Congress \& Exposition (IMECE2012), Texas,USA, 2012

[4] Sahoo, S. \& Das, Jitendra \& Debnath, Bapi., "Rolling element bearing condition monitoring using filtered acoustic emission." International Journal of Electrical and Computer Engineering.vol. 8, no 5, pp. 3560-3567, 2018

[5] Farhat, M.D., Gritli, Y., \& Benrejeb, M., "Fast-ICA for Mechanical Fault Detection and Identification in Electromechanical Systems for Wind Turbine Applications," International Journal of Advanced Computer Science and Applications, vol. 8, no 7, pp. 431-439, 2017

[6] Lee, Daniel D. and Seung, H. Sebastian , "Learning the parts of objects by non-negative matrix factorization," Nature, vol. 401, pp. 788-791, 1999.

[7] O. Hyvarinen, "Independent component analysis: Algorithms and ahallications," Neural Networks, vol. 13, no 4-5, pp. 411-430, 2000.

[8] Ghita, C., Raicu, R. D., \& Pantelimon, B, "Implementation of the FastICA algorithm in sound source separation," 9th International Symposium on Advanced Topics in Electrical Engineering (ATEE), Bucharest, Romania, pp. 19-22, 2015.

[9] Ajami, A., \& Daneshvar, M. , “ Data driven approach for fault detection and diagnosis of turbine in thermal power plant using Independent Component Analysis (ICA)," International Journal of Electrical Power \& Energy Systems, vol. 43, no 1, pp. 728-735, 2012.

[10] Ji, Z. P., \& Zhang, X. J. , “An Integrated Fault Diagnosis Method Based on the ICA-SVM,” Applied Mechanics and Materials, vol. 740, pp. 523-526, 2015.

[11] Wang H, Li R, Tang G, Yuan H, Zhao Q, Cao X. , "A Compound fault diagnosis for rolling bearings method based on blind source separation and ensemble empirical mode decomposition," PLoS One, vol. 9, no 10, pp. 1-13, 2014.

[12] Cichocki, A., Zdunek, R., Phan, A. H., \& Amari, S. I., "Nonnegative matrix and tensor factorizations: applications to exploratory multi-way data analysis and blind source separation," John Wiley \& Sons, 2009.

[13] Norsalina Hassan, Dzati Athiar Ramli, "A Comparative Study of Blind Source Separation for Bioacoustics Sounds based on FastICA, PCA and NMF," Procedia Computer Science, vol. 126, pp. 363-372, 2018.

[14] Févotte, Cédric, Nancy Bertin, and Jean-Louis Durrieu, "Nonnegative matrix factorization with the Itakura-Saito divergence: With application to music analysis," Neural computation, vol. 21, no 3, pp. 793-830, 2009.

[15] Ozerov, Alexey, and Cédric Févotte, "Multichannel nonnegative matrix factorization in convolutive mixtures for audio source separation," IEEE Transactions on Audio, Speech, and Language Processing, vol. 18, no 3, pp. 550-563, 2010.

[16] Liang, L., Shan, L., Liu, F., Niu, B., \& Xu, G. , "Sparse Envelope Spectra for Feature Extraction of Bearing Faults Based on NMF," Applied Sciences, vol. 9, no 4, p. 755, 2019.

[17] Wodecki, J., Zdunek, R., Wyłomańska, A., \& Zimroz, R., "Nonnegative factorization of spectrogram for local damage detection of belt conveyor gearboxes," FAC-PapersOnLine, vol. 50, no 1, pp. 4714-4718, 2017.

[18] Cécilia Damon, A.ntoine Liutkus, Alexandre Gramfort, Slim Essid, "Non-negative matrix factorization for singlechannel EEG artifact rejection," International Conference on Acoustics, Speech, and Signal Processing (ICASSP), Vancouver, Canada, pp. 117-1181, 2013 
[19] Miao, F., Feng, R., \& Wang, X. , "Rotor-Mechanical Coupled Fault Feature Extraction Based on Second-order Blind Identification," 2018 International Symposium on Power Electronics and Control Engineering (ISPECE 2018), Xi'an. China, 2019.

[20] Wang Qinghua, Zhang Youyun, Cai Lei, Zhu Yongsheng, "Fault diagnosis for diesel valve trains based on non-negative matrix factorization and neural network ensemble," Mechanical Systems and Signal Processing, vol. 23, no 5, pp. 1683-1695, 2009

[21] Yong-sheng Yang, An-bo Ming, You-yun Zhang, Yong-sheng Zhu, "Discriminative non-negative matrix factorization (DNMF) and its application to the fault diagnosis of diesel engine," Mechanical Systems and Signal Processing, vol. 95, pp.158-171, 2017.

[22] S. B. Jiang, P. K. Wong, R. Guan, Y. Liang and J. Li, "An Efficient Fault Diagnostic Method for Three-Phase Induction Motors Based on Incremental Broad Learning and Non-Negative Matrix Factorization," in IEEE Access, vol. 7, pp. 17780-17790, 2019.

[23] N. Feng, X. Zhang, Z. Zou, Y. Wang and S. Yi, "Rail health monitoring using acoustic emission technique based on NMF and RVM," 2015 IEEE International Instrumentation and Measurement Technology Conference (I2MTC), Pisa, 2015.

[24] V. Thipsuwanporn, A. Numsamran and M. Leawsoong, "Balance weight fault detection in compressor using FFT algorithm," 12th International Conference on Control, Automation and Systems, Jeju, pp. 798-802, 2012.

[25] A. Advembrio, "Vibration analysis on Compressor Atlas Copco GA 37," Vibration Inspection Section, Gresik, 2017.

\section{BIOGRAPHIES OF AUTHORS}
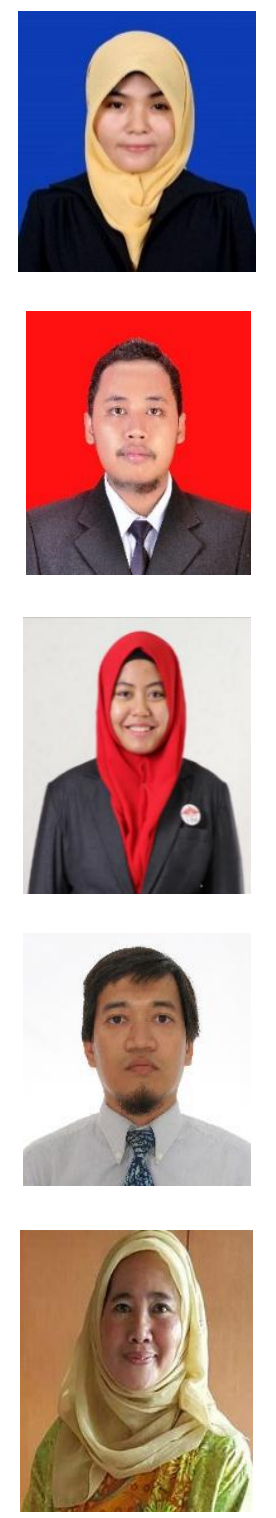

Anindita Adikaputri Vinaya was graduated from the Department of Engineering Physics Institut Teknologi Sepuluh Nopember in 2013. Then, she continued his study in the field of industrial instrumentation in the same department. She earned a master's degree in 2015 and did a research about acoustic signal separation. She currently works as a lecturer at Universitas Internasional Semen Indonesia. Her recent research is focused on the field of reliability and signal processing.

Sefri Yulianto was accepted in the Engineering Management Department, Faculty of Industrial Technology and Agroindustry - Universitas Internasional Semen Indonesia in 2013. During the lecture period, the author was active in various student activities and organizations. The author has also been active in various scientific activities such as the Student Creativity Program. The focus of his research is the processing of acoustic signals.

Qurrotin Ayunina received the bachalor degree (S.T.) in mechanical engineering from Institut Teknologi Sepuluh Nopember (ITS) Surabaya and master degree (M.S.) in mechanical engineering from National Cheng Kung University, Taiwan, in 2012 and 2014, respectively. Her current research area includes renewable energy, thermal sciences, and energy efficiency. Since 2016, she has joined the department of engineering management in Universitas Internasional Semen Indonesia as a lecturer.

Dhany Arifianto completed a bachelor's degree in engineering physics from Institut Teknologi Sepuluh Nopember, Surabaya. He received M.Eng degree in 2002 and Dr.Eng degree in 2005 from Tokyo Institute of Technology. His research interests related to digital signal processing and convex optimization. He has several journal and conference publications in national and international level. He is an active member of Hitachi and IEEE. He has also served as a keynote speaker in several international conferences.

Aulia Siti Aisjah received her Master's degree from the Department of Electrical Engineering, Institut Teknologi Sepuluh Nopember in 1996. She received her doctoral degree in 2007 from the Department of Marine Engineering, Institut Teknologi Sepuluh Nopember. She studied the fields of artificial intelligence and control systems. He received a professor degree in 2016 in the field of Engineering Physics. She currently teaches as a lecturer in the Department of Engineering Physics, Institut Teknologi Sepuluh Nopember. 